\title{
Clinical characteristics and correlates of patients with heart failure with mid-range ejection fraction in southwest Nigeria
}

\author{
Adeseye Akintunde ${ }^{1,2}$ \\ ${ }^{1}$ Department of Medicine, Faculty of Clinical Sciences, Ladoke Akintola University of Technology \\ (LAUTECH) \& LAUTECH Teaching Hospital, Ogbomoso; ${ }^{2}$ Goshen Heart Clinic, Osogbo, Nigeria
}

\begin{abstract}
A new class of Heart Failure (HF) phenotypes, HF with midrange Ejection Fraction (HFmrEF), was recently introduced, but its clinical characteristics and therapeutic distinctiveness are not yet well understood. This study aimed to describe the clinical characteristics, echocardiographic features, and other correlates of HFmrEF in southwest Nigeria. Two hundred and sixty-nine consecutive HF subjects who had echocardiography done in the cardiology clinics of two teaching hospitals were recruited for this study. Clinical parameters such as age, body mass index, waist-hip ratio, and gender were determined. The presence of comorbidities, such as hypertension and diabetes, was also assessed. Statistical
\end{abstract}

Correspondence: Adeseye Akintunde, Goshen Heart Clinic, POB 3238, Osogbo, Nigeria.

Tel.: +234-8033932076

E-mail: aaakintunde@lautech.edu.ng

Key words: Heart failure with mid range ejection fraction; clinical characteristics; Nigeria.

Acknowledgements: To Dr Alamu Olubola for the editorial assistance.

Conflict of interest: The Author declares no conflict of interest.

Availability of data and materials: All data generated or analyzed during this study are included in this published article.

Ethics approval and consent to participate: The Ethics Committee of LAUTECH Teaching Hospital, Ogbomoso approved this study (LTH/019/180). The study is conformed with the Helsinki Declaration of 1964, as revised in 2013, concerning human and animal rights. All patients participating in this study signed a written informed consent form for participating in this study.

Informed consent: Written informed consent was obtained from a legally authorized representative(s) for anonymized patient information to be published in this article.

Received for publication: 13 June 2021.

Revision received: 14 August 2021.

Accepted for publication: 10 September 2021.

This work is licensed under a Creative Commons Attribution NonCommercial 4.0 License (CC BY-NC 4.0).

CCopyright: the Author(s),2020

Licensee PAGEPress, Italy

Annals of Clinical and Biomedical Research 2021; 2:150

doi:10.4081/acbr.2021.150 analysis was done, and $\mathrm{p}<0.05$ was taken as statistically significant. HFmrEF subjects constituted $27.5 \%$ of total cohort, while subjects with HF with preserved Ejection Fraction (HFpEF) and HF with reduced Ejection Fraction (HFrEF) were 29.0\% and $43.5 \%$ respectively. HFmrEF was more likely to be associated with high systolic blood pressure and obesity. The clinical characteristics of HFmrEF were intermediate between those of the other two HF phenotypes. Prevalence of comorbidities, such as anaemia, iron deficiency, pulmonary hypertension, and left ventricular hypertrophy were also intermediate between HFpEF and HFrEF. Atrial fibrillation was commonest among HFmrEF subjects. There was no significant age or gender variation between the three phenotypes. Patients with HFmrEF have clinical and demographic distinctiveness that are often intermediate between HFpEF and HFrEF phenotypes. Further studies of this HF phenotype will help in understanding its therapeutic identity and its prognosis among Africans.

\section{Introduction}

Heart Failure (HF) continues to be a major cause of cardiovascular morbidity and mortality worldwide, contributing significantly to health expenditure. ${ }^{1,2}$ The conventional index of classifying heart failure using ejection fraction has traditionally evolved over the years, ${ }^{3,4}$ and a new class called HF with mid-range Ejection Fraction (HFmrEF) was recently introduced. ${ }^{5,6}$ This class identifies patients whose Left Ventricular Ejection Fraction (LVEF) is between 40 and $49 \%$ in the presence of other clinical features that are compatible with heart failure. HFmrEF has been shown in several studies among Caucasians to have clinical characteristics and prognostic factors that are intermediate between HF with preserved Ejection Fraction (HFpEF) and HF with reduced Ejection Fraction (HFrEF). ${ }^{7-10}$ Other authors have also demonstrated that there are similarities, in many fronts, of HFmrEF to HFpEF in terms of prognosis, and that haemodynamic transition among the three heart failure phenotypes is possible. ${ }^{11}$ The traditional method of classifying heart failure phenotypes has led to an evolving body of evidence in prognosis, morbidity and mortality, and cardiovascular pharmacology in heart failure management. ${ }^{12-15}$ Most available evidence have shown a relatively better prognosis in $\mathrm{HFrEF}$ compared to HFpEF. ${ }^{14-16}$ Many clinical trials in HFpEF subjects have resulted in disappointing outcomes, and the relative lack of evidence-based guideline on the cardiovascular pharmacology of HFpEF, including the recently discovered drugs such as Angiotensin Receptor Blocker-Neprilysin Inhibitors (ARNIs), has been the major bane in its treatment over the years. ${ }^{17-19}$

The first definition of HFmrEF was presented in 2013 by the American College of Cardiology/American Heart Association guideline, and it was defined as the presence of symptoms of heart failure and a LVEF of 40-49\%, while in 2016 it was further specified in the European Society of Cardiology (ESC) guideline for heart failure. ${ }^{20,21}$ Other authors have shown that patients with 
HFmrEF have clinical profile and prognosis that are closer to those of patients with HFpEF than those of patients with HFrEF, with certain distinctions. Currently available treatment modalities for $\mathrm{HFpEF}$ focus on aggressive management of comorbidities and symptomatic control; however, whether HFmrEF patients represent a unique and dynamic HF group that may benefit from targeted therapies known to be beneficial in patients with HFrEF, such as neurohormonal blockade, requires further study. It will therefore be necessary to further characterize this new subgroup with a view to further determining whether they will benefit from available therapeutic management in $\mathrm{HF}$, like $\mathrm{HFrEF}$, or they will require specific management strategies to reduce the high cardiovascular mortality and morbidity. ${ }^{21}$

Analysis of large heart failure cohorts, such as Organized Program to Initiate Lifesaving Treatment in Hospitalized Patients with Heart Failure (OPTIMIZE-HF) and Acute Decompensated Heart Failure (ADHERE-HF) registries, revealed that demographic characteristics, symptom profile, comorbidities, laboratory values, and short-term outcomes of patients with LVEF between $40 \%$ and $50 \%$ were closer to those of patients with HFpEF. ${ }^{22,23}$ Other studies have indicated that HFpEF and HFrEF have distinct pathophysiologic mechanisms, but the underlying pathophysiologic mechanisms in HFmrEF remain unclear. The 2016 ESC guidelines suggested that patients with HFmrEF likely have mild systolic dysfunction as well as diastolic dysfunction. ${ }^{21}$

However, the clinical characteristics and correlates of HFmrEF have not been well described among Nigerians with heart failure. This study therefore aimed to describe the clinical, demographic, and laboratory characteristics, and clinical correlates of heart failure subjects with mid-range ejection fraction in southwest Nigeria.

\section{Materials and Methods}

This was a retrospective study. Data was obtained from a registry of heart failure subjects who were seen and had echocardiography in the cardiology clinics of two teaching hospitals: Ladoke Akintola University of Technology Teaching Hospital, Ogbomoso, and Bowen University Teaching Hospital, Ogbomoso, Nigeria. Ethical approval was obtained from Ladoke Akintola University of Technology Teaching Hospital, Ogbomoso, Nigeria. Informed written consent was also obtained from all study participants.

Heart failure was diagnosed by the presence of typical symptoms and signs according to validated guidelines. ${ }^{21}$ All subjects included in this study also had echocardiography, among other investigations. The relevant clinical and demographic parameters were obtained from the case records of the patients, and they include age, gender, occupation, presence of hypertension or diabetes mellitus and the duration of diagnosis of these conditions, drugs being used, and history of smoking and alcohol intake. Relevant examination findings were noted, including cardiovascular examination with the average of three blood pressure readings measured.

Transthoracic echocardiography was performed according to the American Society of Echocardiography guideline. ${ }^{24}$ The patient was placed in the left lateral decubitus position, and the left parasternal views (long and short axis views) were examined. The left apical view for the standard four-chamber view, the short axis view, and the subcostal view were also assessed. The following parameters were obtained: interventricular septal thickness in diastole, posterior wall thickness in diastole, left ventricular end diastolic and end systolic internal dimensions, right ventricular dimension, aortic root dimension, aortic cusp separation, and left atrial dimension. The ejection fraction and fractional shortening were determined using the Teicholz formula. Doppler echocardiography was done, and the left and right ventricular inflow velocities, including mitral Early (E) and Late (A) velocities, and tricuspid E and $\mathrm{A}$ velocities, were determined. The ratio of $\mathrm{E} / \mathrm{A}$ was determined, and the deceleration time was also obtained. Diastolic dysfunction was defined based on conventional guideline. Other parameters obtained from echocardiography include left ventricular mass, left ventricular mass index, and relative wall thickness. Tricuspid Annular Plane Systolic Excursion (TAPSE) was used to define right ventricular systolic function. Other investigations that were done include full blood count; serum electrolytes, urea and creatinine; lipid profile; and electrocardiography. Left ventricular hypertrophy was defined using the left ventricular mass index. Atrial fibrillation was defined as the absence of $P$ waves on routine 12-lead ECG. Pulmonary hypertension (PAP $>25 \mathrm{mmHg}$ ) was defined using estimated pulmonary arterial systolic pressure which was gotten through the addition of the tricuspid regurgitant velocity pressure gradients added to the estimated right atrial pressure. Some of the heart failure subjects had full iron status parameters determined, including total serum iron, ferritin, transferrin, and transferrin saturation. Hypertension was defined as blood pressure $>140 / 90 \mathrm{mmHg}$ measured at least two times or patient already on antihypertensive medications. Anaemia was defined as haemoglobin levels $<12.0 \mathrm{~g} / \mathrm{dL}$ in women and $<13.0 \mathrm{~g} / \mathrm{dL}$ in men according to WHO criteria. Total serum iron and total iron binding capacity were measured using the colometric kits from fortress diagnostics (Product code BXC0234) Iron deficiency was defined as low total serum iron and transferrin saturation $<20 \%$ as it is a better marker of iron status than ferritin, a pro-inflammatory marker. ${ }^{25,26}$ Kidney dysfunction was defined as estimated glomerular filtration rate $<60 \mathrm{~mL} / \mathrm{min} / 1.732 \mathrm{~m}^{2}$.

Statistical analysis was done with the Statistical Package for Social Sciences (SPSS) Chicago III Version 21.0. P $<0.05$ was taken as statistically significant. Quantitative data were summarized as means \pm standard deviations, while qualitative data were expressed in frequencies and percentages. Group comparison was done with the aid of Student's t test, analysis of variance and Chi square as appropriate, with appropriate correction.

\section{Results}

Two hundred and sixty-nine heart failure subjects were included in this analysis. They all had complete records and had echocardiography done by the researcher. They were included as heart failure subjects who consecutively had echocardiography performed as part of their routine investigation and treatment. Using the definition of heart failure phenotypes based on left ventricular ejection fraction, $78(29.0 \%), 74(27.5 \%)$ and $117(43.5 \%)$ subjects were classified into HFpEF, HFmrEF and HFrEF groups respectively as shown in Figure 1.

The clinical and demographic characteristics of study participants based on heart failure phenotypes are shown in Table 1. Age and gender distributions were similar among the various heart failure phenotypes. Even though subjects with HFmrEF were the eldest, this finding was not statistically significant. Systolic blood pressure in HFpEF $(134.6 \pm 24.0)$, HFmrEF $(130.8 \pm 26.1)$ and HFrEF (122.9 \pm 24.2$)$ were significantly higher between groups; $p$ $<0.05$ ). Mean body mass index was also significantly higher between the groups with it being highest among HFpEF (28.8 $\pm 7.9)$ and lowest among HFrEF $\left(23.6 \pm 6.0 \mathrm{~kg} / \mathrm{m}^{2}\right)$ subjects.

Diastolic blood pressure, packed cell volume and waist-hip 
ratio were higher among HFpEF subjects compared to others, but this was not of statistical significance. Mean fasting blood sugar was highest among HFrEF subjects, but this also was not statistically significant. The likelihood of being in advanced heart failure (New York Heart Association stage IV) was significantly more with HFrEF subjects compared to others. The proportion of participants in NYHA IV was higher among HFrEF subjects compared to HFmrEF and HFpEF subjects $(46.2 \%$ vs. $28.4 \%$ vs. $10.3 \%$ respectively, $\mathrm{p}<0.05)$. Smoking and alcohol intake were not significantly different among the three groups. Serum urea was significantly higher among HFmrEF $(19.6 \pm 26.8 \mathrm{mmol} / \mathrm{L})$ subjects compared to HFpEF $(3.36 \pm 1.81 \mathrm{mmol} / \mathrm{L})$ and $\mathrm{HFrEF}(14.1 \pm 18.5$ $\mathrm{mmol} / \mathrm{L})$ subjects as shown in Table $1 . \mathrm{p}<0.05)$. Serum creatinine was highest among HFrEF subjects, but the difference was not significant statistically. Serum atrial natriuretic peptide was also shown to be highest among HFmrEF subjects, and average value was similar to what was obtained among HFpEF subjects.

Table 2 shows the distribution of clusters of comorbidities in the study population. Hypertension was more prevalent in $\mathrm{HFpEF}$ $(92.3 \%)$ and HFmrEF (89.2\%) subjects compared to HFrEF(78.6\%) subjects. Similarly, diabetes mellitus had higher prevalence among HFpEF and HFmrEF subjects as shown in Table 2.Among HFrEF, HFmrEF and HFpEF subjects respectively, the prevalence of pulmonary hypertension was $66.7 \%$ in HFrEF compared to $27.0 \%$ in HFmrEF and $5.1 \%$ in HFpEF patients $(\mathrm{p}<0.05)$. Anaemia was documented in $91.5 \%$ of HFrEF compared to $73.0 \%$ in HFmrEF and $55.1 \%$ in HFpEF patients $(p<0.05)$. Kidney dysfunction was found in $32.5 \%$ of HFrEF patients compared to $27.0 \%$ in HFmrEF patients and $3.85 \%$ in HFpEF patients ( $p$ $<0.05$ ). The prevalence of aortic root dilatation was more common in HFrEF (27.4\%) compared to $16.2 \%$ in HFmrEF and $17.9 \%$ in HFpEF patients. Low transferrin was documented in $70.1 \%$ of HFrEF and $32.7 \%$ of patients with HFpEF. Similarly, the prevalence of left ventricular chaber hypertrophy on echocardiography was $70.1 \%$ in HFrEF compared to $64.9 \%$ in HFmrEF and $44.9 \%$ in HFpEF subjects. In the same vein intra-cardiac clots was more common among subjects with HFrEF compared to other groups, $\mathrm{p}<0.05$. Obesity (body mass index $>30 \mathrm{~kg} / \mathrm{m}^{2}$ ) was significantly more associated with HFpEF subjects, its prevalence being $35.9 \%$ in HFpEF compared to $20.3 \%$ in HFmrEF subjects and $14.5 \%$ among HFrEF subjects $(\mathrm{p}<0.05)$ as shown in Table 2 . Atrial fibrillation and iron deficiency occurred more among HFmrEF compared to HFpEF and HFrEF subjects as shown in Table 2 also.

The echocardiographic parameters among the various heart failure phenotypes are shown in Table 3. Left ventricular internal dimensions and wall measurements were significantly higher among HFrEF subjects compared to other heart failure phenotypes. Left ventricular internal dimension in diastole was significantly highest among subjects with HFrEF as shown in Table 3 compared to other groups. Similarly, left ventricular internal dimension in systole was significantly higher among HFrEF than the other groups. As shown also in Table 3. Posterior wall thickness in diastole was also higher among HFrEF subjects than other heart failure phenotypes, but this was not of statistical significance. Interventricular septal thickness in diastole was higher among HFmrEF patients compared to other groups. For HFrEF, HFmrEF, and HFpEF subjects respectively, right ventricular internal dimension, left atrial dimension, left ventricular mass and left ventricular mass indexed to height ${ }^{27}$ were significantly higher among $\mathrm{HFrEF}$ than HFmrEF and also HFpEF subjects, as shown in Table 3.

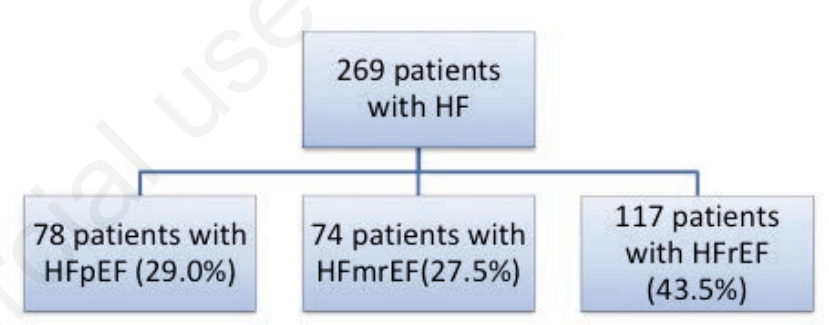

Figure 1. The distribution of heart failure phenotypes based on left ventricular ejection fraction. HF, heart failure; $\mathrm{HFpEF}$, heart failure with preserved ejection fraction; HFmrEF, heart failure with mid-range ejection fraction; HFrEF, heart failure with reduced ejection fraction

Table 1. Clinical and demographic variables among the heart failure phenotypes.

\begin{tabular}{|c|c|c|c|c|}
\hline Variable & HFPEF & HFmrEF & HFrEF & P value \\
\hline Age (years) & $60.0 \pm 15.1$ & $63.8 \pm 17.3$ & $61.7 \pm 15.5$ & 0.509 \\
\hline Males(n) & $35(44.9 \%)$ & $38(51.4 \%)$ & $66(56.4 \%)$ & 0.498 \\
\hline SBP (mmHg) & $134.6 \pm 24.0$ & $130.8 \pm 26.1$ & $122.9 \pm 24.2$ & $0.020^{*}$ \\
\hline $\mathrm{DBP}(\mathrm{mmHg})$ & $80.4 \pm 12.6$ & $81.2 \pm 15.8$ & $78.1 \pm 18.6$ & 0.478 \\
\hline WHR & $0.96 \pm 0.07$ & $0.94 \pm 0.10$ & $0.93 \pm 0.07$ & 0.100 \\
\hline PCV (\%) & $35.1 \pm 4.4$ & $32.2 \pm 7.3$ & $31.3 \pm 6.4$ & 0.186 \\
\hline BMI $\left(\mathrm{kg} / \mathrm{m}^{2}\right)$ & $28.8 \pm 7.9$ & $25.2 \pm 6.0$ & $23.6 \pm 6.0$ & $0.001^{*}$ \\
\hline FBS $(\mathrm{mmol} / \mathrm{l})$ & $5.2 \pm 0.84$ & $6.7 \pm 1.2$ & $8.3 \pm 1.2$ & 0.169 \\
\hline Alcohol (n) & $10(12.8 \%)$ & $22(29.7 \%)$ & $33(28.2 \%)$ & 0.05 \\
\hline Smoking (n) & $5(6.4 \%)$ & $8(10.8 \%)$ & $37(31.6 \%)$ & 0.106 \\
\hline NYHA IV (n) & $8(10.3 \%)$ & $21(28.4 \%)$ & $54(46.2 \%)$ & $0.004^{*}$ \\
\hline Urea (mmol/l) & $3.36 \pm 1.81$ & $19.6 \pm 26.8$ & $14.1 \pm 18.5$ & $0.018^{*}$ \\
\hline Creatinine $(\mu \mathrm{mol} / \mathrm{l})$ & $84.4 \pm 11.6$ & $128.3 \pm 70.8$ & $156.1 \pm 168.6$ & 0.228 \\
\hline Heart rate (beats/min) & $70.0 \pm 30.2$ & $90.0 \pm 24.0$ & $105.3 \pm 18.1$ & $0.000^{*}$ \\
\hline Atrial natriuretic peptides (pg/ml) & $166.0 \pm 72.0$ & $168.2 \pm 43.1$ & $152.9 \pm 56.7$ & 0.571 \\
\hline
\end{tabular}

*statistically significant. Key to table: BMI, body mass index; DBP, diastolic blood pressure; FBS, fasting blood sugar; HFmrEF, heart failure with mid-range ejection fraction; HFrEF, heart failure with reduced ejection fraction; HFpEF, heart failure with preserved ejection fraction; NYHA, New York Heart Association; SBP, systolic blood pressure; PCV, packed cell volume; WHR, waist-hip ratio. 


\section{Discussion}

This study examined the clinical characteristics of a recently introduced heart failure phenotype that has hitherto been without much recognition. Since the available guidelines are based on the previously available classification upon which studies were performed, it is very important to appropriately describe the clinical syndrome of subjects with heart failure with mid-range ejection fraction in order to identify possible therapeutic modalities and inclusions in clinical trials for heart failure pharmacotherapy. ${ }^{27}$ This study revealed that HFmrEF subjects had features that were mostly intermediate between the features of the two major heart failure phenotypes (heart failure with reduced ejection fraction and heart failure with preserved ejection fraction) that had been earlier described. There are however some major clinical and biochemical distinctive features of HFmrEF, with subtle differences in this cohort of Africans, compared to other races that have been described in the literature.

\section{Epidemiology of heart failure with mid-range ejection fraction}

The frequency of HFmrEF in this cohort was $27.5 \%$. HFpEF accounted for $29.0 \%$ while HFrEF accounted for $43.5 \%$ of the total study population. This suggests that at least a quarter of all heart failure subjects in this environment may be described to have the recently classified heart failure phenotype-HFmrEF. The frequency of HFmrEF reported in this study is higher than what was reported in a longitudinal review of patients admitted for heart failure from the cardiac unit of Komfo Anokye Teaching Hospital, Ghana, between 2009 and 2013, where HFmrEF accounted for $17.8 \%$, and $59 \%$ and $23.2 \%$ had HFpEF and HFrEF respectively. ${ }^{28}$ The prevalence found in this study is similarly higher than what has been reported from large prospective and retrospective heart failure registries among Caucasians. In the Chronic Heart Failure Analysis and Registry in the Tohoku District 2 (CHART-2) registry, $17.1 \%$ of participants were shown to have HFmrEF, while $7.5 \%$ were documented to have HFmrEF in the Practice Innovation

Table 2. Cluster of comorbidities and associated clinical conditions with heart failure phenotypes.

\begin{tabular}{|c|c|c|c|c|}
\hline Variable & HFpEF $(n=78)$ & HFmrEF $(n=74)$ & HFrEF ( $n=117)$ & P value \\
\hline Hypertension & $72(92.3 \%)$ & $66(89.2 \%)$ & $92(78.6 \%)$ & 0.094 \\
\hline DM & $8(10.3 \%)$ & $8(10.8 \%)$ & $6(5.1 \%)$ & 0.094 \\
\hline Pulmonary hypertension & $4(5.1 \%)$ & $20(27.0 \%)$ & $78(66.7 \%)$ & $0.000 *$ \\
\hline Anaemia & $43(55.1 \%)$ & $54(73.0 \%)$ & $107(91.5 \%)$ & $0.000^{*}$ \\
\hline $\mathrm{eGFR}<30 \mathrm{ml} / \mathrm{min}$ & $3(3.85 \%)$ & $20(27.0 \%)$ & $38(32.5 \%)$ & $0.000 *$ \\
\hline Aortic root dilatation & $14(17.9 \%)$ & $12(16.2 \%)$ & $32(27.4 \%)$ & $0.001^{*}$ \\
\hline Iron deficiency & $19(24.4 \%)$ & $35(47.3 \%)$ & $54(46.2 \%)$ & $0.000 *$ \\
\hline Low transferrin & $24(30.8 \%)$ & $39(52.7 \%)$ & $82(70.1 \%)$ & $0.000^{*}$ \\
\hline $\mathrm{LVH}$ & $35(44.9 \%)$ & 48 (64.9\%) & $82(70.1 \%)$ & $0.000^{*}$ \\
\hline Obesity (BMI >30kg/m²) & $28(35.9 \%)$ & $15(20.3 \%)$ & $17(14.5 \%)$ & $0.000^{*}$ \\
\hline Atrial fibrillation & $0(0 \%)$ & $7(9.5 \%)$ & $7(6.0 \%)$ & $0.000 *$ \\
\hline Intra-cardiac clots & $1(1.3 \%)$ & $9(12.2 \%)$ & $44(37.6 \%)$ & $0.000 *$ \\
\hline
\end{tabular}

*statistically significant. Key to table: BMI, body mass index; DM, diabetes mellitus; eGFR, estimated glomerular filtration rate; HFmrEF, heart failure with mid-range ejection fraction; HFpEF, heart failure with preserved ejection fraction; HFrEF, heart failure with reduced ejection fraction; LVH, left ventricular chamber hypertrophy.

Table 3. Echocardiographic parameters among various heart failure phenotypes.

\begin{tabular}{|c|c|c|c|c|}
\hline Variable & HFpEF & HFmrEF & HFrEF & P value \\
\hline LVDD (mm) & $44.4 \pm 8.4$ & $53.5 \pm 11.6$ & $60.6 \pm 10.1$ & $0.000^{*}$ \\
\hline LVSD (mm) & $30.4 \pm 5.0$ & $36.0 \pm 14.0$ & $49.4 \pm 12.7$ & $0.000 *$ \\
\hline PWTd (mm) & $11.8 \pm 1.6$ & $12.5 \pm 2.6$ & $12.6 \pm 2.1$ & 0.776 \\
\hline IVSd (mm) & $12.6 \pm 2.1$ & $13.6 \pm 2.7$ & $12.7 \pm 2.1$ & 0.102 \\
\hline RVD (mm) & $26.4 \pm 3.7$ & $29.4 \pm 5.1$ & $32.6 \pm 4.6$ & $0.000^{*}$ \\
\hline $\mathrm{LAD}(\mathrm{mm})$ & $40.4 \pm 5.9$ & $45.6 \pm 15.3$ & $51.7 \pm 7.1$ & $0.000^{*}$ \\
\hline $\mathrm{AOD}(\mathrm{mm})$ & $33.3 \pm 3.4$ & $32.4 \pm 3.8$ & $32.2 \pm 5.1$ & 0.590 \\
\hline $\mathrm{ACS}(\mathrm{mm})$ & $18.1 \pm 2.3$ & $17.6 \pm 3.4$ & $18.0 \pm 3.3$ & 0.420 \\
\hline $\mathrm{EF}(\mathrm{mm})$ & $57.3 \pm 5.7$ & $43.1 \pm 2.2$ & $33.3 \pm 4.2$ & $0.000 *$ \\
\hline TAPSE (mm) & $20.4 \pm 3.9$ & $16.9 \pm 5.6$ & $14.5 \pm 3.4$ & $0.000^{*}$ \\
\hline RWT (mm) & $0.52 \pm 0.06$ & $0.47 \pm 0.12$ & $0.41 \pm 0.09$ & $0.000^{*}$ \\
\hline LVM (mm) & $196.4 \pm 70.0$ & $310.8 \pm 142.1$ & $360.7 \pm 96.7$ & $0.000^{*}$ \\
\hline LVM ht.2.7 & $53.7 \pm 20.3$ & $73.3 \pm 45.1$ & $95.4 \pm 35.3$ & $0.000 *$ \\
\hline
\end{tabular}

* statistically significant. Key to table: ACS, aortic cusp separation; EF, ejection fraction; HFmrEF, heart failure with mid- range ejection fraction $\mathrm{HFpEF}$, heart failure with preserved ejection fraction; HFrEF, heart failure with reduced ejection fraction; IVSd, interventricular septal thickness in diastole; LAD, left atrial dimension; LVDD, left ventricular internal dimension in diastole; LVM, left ventricular mass; LVMht.2.7, left ventricular mass index; LVSD, left ventricular internal dimension in systole; PWTd, posterior wall thickness in diastole; RVD, right ventricular dimension; RWT, relative wall thickness; TAPSE, tricuspid annular plane systolic excursion. 
and Clinical Excellence (PINNACLE) study. ${ }^{29,30}$ The prevalence of HFmrEF in a UK population was reported to be $21 \% .{ }^{31}$ Studies in the United States have suggested that the percentage of the HF population that falls into the HFmrEF category is between $13 \%$ and $24 \%$, suggesting that approximately 1.6 million people in the United States have HFmrEF. ${ }^{29,32,33}$ Therefore, it can be concluded that up to about a quarter of the heart failure population worldwide have the recently classified heart failure phenotype-HFmrEF. The variations in the prevalence of HFmrEF worldwide may be related to many factors. The burden of risk factors for heart failure varies significantly worldwide, with hypertension and cardiomyopathies being the major risk factors in Africa, whereas coronary artery disease predominates among Caucasians. This variation in the prevalence across the regions of the world may also be related to other factors, including diagnostic potentials and the known transition between the various heart failure phenotypes. It has been a major scientific question whether HFmrEF is a distinct heart failure phenotype or a transition between HFpEF and HFrEF. Nonetheless, a better understanding of its profile will allow better characterization and follow-up, with a view to further describing specific therapeutic strategies.

Studies have shown that bidirectional LVEF transition occurs in the course of heart failure, and this may lead to misclassification and use of inappropriate therapy. In these studies, overall, 39\% of HFpEF patients had LVEF $<50 \%$, and $39 \%$ of HFrEF patients had LVEF $\geq 50 \%$ at some point after diagnosis. Decreases in LVEF were associated with reduced survival, and increases with improved survival. Up to $25 \%$ of treated HFrEF patients showed improvement in LVEF. $^{34}$

\section{Clinical characteristics of Nigerians with heart failure with mid-range ejection fraction}

The true clinical characteristics of HFmrEF have been poorly described over the years, as most registry and clinical trials excluded subjects with the phenotype. Describing the true clinical status of these patients will enhance the general understanding, prognosis, and associations of HFmrEF. ${ }^{35}$

This study revealed that majority of HFmrEF subjects had clinical characteristics that were intermediate between those of HFpEF and HFrEF subjects. Gender distribution, systolic blood pressure, diastolic blood pressure, packed cell volume, fasting blood sugar, urea and creatinine were mostly intermediate between values obtained for HFpEF and HFrEF subjects. Mean heart rate and body mass index were also intermediate between those of the other two heart failure phenotypes as shown in Table 1. HFmrEF subjects were older in this cohort compared to the two other types.

Our study also revealed that the prevalence of many comorbidities and associated clinical conditions in HFmrEF were also intermediate between what was obtained for HFrEF and HFpEF. These comorbidities and conditions include systemic hypertension, pulmonary hypertension, anaemia, kidney dysfunction, left ventricular hypertrophy, obesity, and intra-cardiac clots. Some of the clinical characteristics were more similar to those of HFpEF subjects than to HFrEF subjects. Those characteristics include systolic blood pressure, diastolic blood pressure, and the prevalence of hypertension, diabetes mellitus and obesity. HFmrEF subjects were more similar to HFrEF subjects in the frequency of pulmonary hypertension, kidney dysfunction, iron deficiency, and left ventricular hypertrophy. These findings are consistent with findings from other studies that have shown that features of HFmrEF are intermediate between the two other phenotypes, and that they are more closely similar to $\mathrm{HFpEF}$ in many respects, including demographic characteristics, symptom profile, comorbidities, laboratory values, and short-term outcomes of patients. ${ }^{36,37}$

\section{Prognostic clinical and echocardiographic factors among HFmrEF subjects}

The clinical course of heart failure can be determined by many factors. Many biomarkers and echocardiographic parameters have been shown to be conventionally related to prognosis in heart failure care. They include ejection fraction, fractional shortening, Tei index, left ventricular mass index, and cardiac natriuretic peptides (such as atrial natriuretic peptide and brain natriuretic peptides), among others. Serum atrial natriuretic peptide values were similar between HFpEF and HFmrEF subjects in this study. HFpEF was associated with significantly higher mean serum ANP levels compared to other heart failure phenotypes, and this is related to the high risk of the morbidity and mortality associated with HFpEF. ${ }^{38}$

Majority of the clinical determinants of prognosis in HFmrEF subjects were more closely related to HFrEF. They include the prevalence of anaemia, iron deficiency, pulmonary hypertension, atrial fibrillation, and kidney dysfunction. The highest prevalence of atrial fibrillation was recorded among HFmrEF subjects in this study, and this also reflects the inherently poor morbidity associated with this group of patients. Thus, it can be suggested that HFmrEF has prognosis similar to HFrEF in this population and should therefore be addressed to reduce the burden of heart failure in the general population.

\section{Echocardiographic variations among the various heart failure phenotypes}

This study also revealed that majority of the echocardiographic parameters of HFmrEF were intermediate between HFpEF and HFrEF phenotypes. The mean left ventricular chamber wall dimensions, left and right ventricular systolic function, and left atrial and right ventricular dimensions were mostly intermediate between the other two heart failure phenotypes. It thus suggests the intermediate position of HFmrEF between the other phenotypes. Relative wall thickness and left ventricular mass index values were also in-between those of the other two phenotypes. Some of these echocardiographic parameters, such as left ventricular mass, left ventricular internal dimensions, ejection fraction, and tricuspid annular plane systolic excursion, have important prognostic characteristics. ${ }^{39,40}$

The role of echocardiography in risk stratification of heart failure patients has been a matter of interest. Some authors have reported that among African heart failure subjects, echocardiographic parameters such as left atrial size, heart rate, and measures of left ventricular hypertrophy, such as posterior wall dimensions and left ventricular mass, were predictive of prognosis in heart failure. ${ }^{40}$

Limitations of this study include its hospital-based nature and the fact that it was a retrospective review that may not have completely captured the true clinical characteristics of patients, especially considering that heart failure may transit from one phenotype to another. The relatively small number of registry participants can also limit the interpretation of the study.

\section{Conclusions}

This study revealed that HFmrEF subjects had features that were mostly intermediate between HFrEF and HFpEF subjects. They were closely related to HFpEF subjects with respect to sys- 
tolic and diastolic blood pressures, and prevalence of hypertension, diabetes, and obesity. They were however more closely related to HFrEF subjects in specific areas such as age and presence of comorbidities such as anaemia, iron deficiency, pulmonary hypertension, intra-cardiac clots, kidney dysfunction, and left ventricular hypertrophy. Further description of this new heart failure phenotype will lead to better understanding of its pathology and design of appropriate clinical trials for its specific pharmacology.

\section{References}

1. Ponikowski P, Voors AA, Anker SD, et al. ESC Scientific Document Group. 2016. ESC Guidelines for the diagnosis and treatment of acute and chronic heart failure: the Task Force for the diagnosis and treatment of acute and chronic heart failure of the European Society of Cardiology (ESC) developed with the special contribution of the Heart Failure Association (HFA) of the ESC. Eur Heart J 2016;37:2129-2200.

2. Casey JDE, Colvin MM, Drazner MH, et al. 2016 ACC/AHA/HFSA focused update on new pharmacological therapy for heart failure: an update of the 2013 ACCF/AHA guideline for the management of heart failure: a report of the American College of Cardiology/American Heart Association Task Force on Clinical Practice Guidelines and the Heart Failure Society of America. J Am Coll Cardiol 2016;68:147688 .

3. Solomon SD, Anavekar N, Skali H, et al. Influence of ejection fraction on cardiovascular outcomes in a broad spectrum of heart failure patients. Circulation 2005;112:3738-3744.

4. Coles AH, Fisher K, Darling C, et al. Long-term survival for patients with acute decompensated heart failure according to ejection fraction findings. Am J Cardiol 2014;114:862-8.

5. Hsu JJ, Ziaeian B, Fonarow GC. Heart failure with mid-range (borderline) ejection fraction: Clinical implications and future directions. JACC Heart Fail 2017;5:763-71.

6. Lopatin Y. Heart failure with mid-range ejection fraction and how to treat it. Card Fail Rev 2018;4:9-13.

7. Lauritsen J, Gustafsson F, Abdulla J. Characteristics and longterm prognosis of patients with heart failure and mid-range ejection fraction compared with reduced and preserved ejection fraction: a systematic review and meta-analysis. ESC Heart Fail 2018;5:685-94.

8. Mesquita ET, Barbetta LMDS, Correia ETO. Heart failure with mid-range ejection fraction - state of the art. Arq Bras Cardiol 2019;112:784-90.

9. Nadar SK, Tariq O. What is heart failure with mid-range ejection fraction? A new subgroup of patients with heart failure. Card Fail Rev 2018;4:6-8.

10. Tsuji K, Sakata Y, Nochioka K, et al. Characterization of heart failure patients with mid-range left ventricular ejection fraction-a report from the CHART-2 Study. Eur J Heart Fail 2017;19:1258-69.

11. Triposkiadis F, Butler J, Abboud FM, et al. The continuous heart failure spectrum: moving beyond an ejection fraction classification. Eur Heart J 2019;40:2155-63.

12. Kao DP, Lewsey JD, Anand IS, et al. Characterization of subgroups of heart failure patients with preserved ejection fraction with possible implications for prognosis and treatment response. Eur J Heart Fail 2015;17:925-935.

13. Rickenbacher P, Kaufmann BA, Maeder MT, et al. For the TIME-CHF Investigators. Heart failure with mid-range ejection fraction: a distinct clinical entity? Insights from the Trial of Intensified versus standard Medical therapy in Elderly patients with Congestive Heart Failure (TIME-CHF). Eur J Heart Fail 2017;19:1586-96.

14. Meta-analysis Global Group in Chronic Heart Failure (MAGGIC). The survival of patients with heart failure with preserved or reduced left ventricular ejection fraction: an individual patient data meta-analysis. Eur Heart J 2012;33:1750-7.

15. Hernandez AF, Hammill BG, O'Connor CM, et al. Clinical effectiveness of beta-blockers in heart failure: findings from the OPTIMIZE-HF (Organized Program to Initiate Lifesaving Treatment in Hospitalized Patients with Heart Failure) Registry. J Am Coll Cardiol 2009;53:184-92.

16. Dharmarajan K, Rich MW. Epidemiology, pathophysiology and prognosis of heart failure in older adults. Heart Fail Clin 2017;13:417-26.

17. Biglane JB, Becnel MF, Ventura HO, Krim SR. Pharmacologic therapy for heart failure with reduced ejection fraction: Closing the gap between clinical guidelines and practice. Prog Cardiovasc Dis 2017;60:187-97.

18. Lund LH, Claggett B, Liu J, et al. Heart failure with mid-range ejection fraction in CHARM: characteristics, outcomes and effect of candesartan across the entire ejection fraction spectrum. Eur J Heart Fail 2018;20:1230-39.

19. Wang TJ, Evans JC, Benjamin EJ, et al. Natural history of asymptomatic left ventricular systolic dysfunction in the community. Circulation 2003;108:977-82.

20. Ponikowski P, Voors AA, Anker SD, et al. 2016 ESC Guidelines for the diagnosis and treatment of acute and chronic heart failure: The Task Force for the diagnosis and treatment of acute and chronic heart failure of the European Society of Cardiology (ESC). Developed with the special contribution of the Heart Failure Association (HFA) of the ESC. Eur J Heart Fail 2016;18:891-975.

21. Fonarow GC, Stough WG, Abraham WT, et al. Characteristics, treatments, and outcomes of patients with preserved systolic function hospitalized for heart failure: a report from the OPTIMIZE-HF Registry. J Am Coll Cardiol 2007;50:768-77.

22. Sweitzer NK, Lopatin M, Yancy CW, et al. Comparison of clinical features and outcomes of patients hospitalized with heart failure and normal ejection fraction ( $>$ or $=55 \%$ ) versus those with mildly reduced $(40 \%$ to $55 \%)$ and moderately to severely reduced $(<40 \%)$ fractions. Am J Cardiol 2008;101:1151-6.

23. Abraham WT, Fonarow GC, Albert NM, et al. Predictors of inhospital mortality in patients hospitalized for heart failure: Insights from the Organized Program to Initiate Lifesaving Treatment in Hospitalized Patients with Heart Failure (OPTIMIZE-HF) J Am Coll Cardiol 2008;52:347-56.

24. Butler J, Fonarow GC, Zile MR, et al. Developing therapies for heart failure with preserved ejection fraction: current state and future directions. J Am Coll Cardiol HF 2014;2:97-112.

25. Van Veldhuisen DJ, Anker SD, Ponikowski P, Macdougali IC. Anaemia and iron deficiency in heart failure: mechanisms and therapeutic approaches. Nat Rev Cardiol 2011;8:485-93

26. Okonko DO, Grzeslo A, Witkowski T, et al. Effect of intravenous iron sucrose on exercise tolerance in anemic and nonanemic patients with symptomatic chronic heart failure and iron deficiency FERRIC-HF: a randomized controlled observer blinded trial. J Am Coll Cardiol 2008;51:103-12.

27. Bonsu KO, Owusu IK, Buabeng KO, et al. Clinical characteristics and prognosis of patients admitted for heart failure: a 5 year retrospective study of African patients. Int J Cardiol 2017;238:128-35. 
28. Shiga T, Suzuki A, Haruta S, et al. Clinical characteristics of hospitalized heart failure patients with preserved, mid-range, and reduced ejection fractions in Japan. ESC Heart Fail 2019;6:475-86.

29. Webb J, Drapper J, Fovargue L, et al. Is heart failure with midrange ejection fraction (HFmrEF) a distinct clinical entity or an overlap group? IJC Heart Vasculature 2018;21:1-6.

30. Kapoor JR, Kapoor R, Ju C, Heidenreich PA, et al. Precipitating clinical factors, heart failure characterization, and outcomes in patients hospitalized with heart failure with reduced, borderline, and preserved ejection fraction. J Am Coll Cardiol HF 2016;4:464-72.

31. Coles AH, Tisminetzky M, Yarzebski J, et al. Magnitude of and prognostic factors associated with 1-year mortality after hospital discharge for acute decompensated heart failure based on ejection fraction findings. J Am Heart Assoc 2015;4:e002303.

32. Triposkiadis F, Butler J, Abboud FM, et al. The continuous heart failure spectrum: moving beyond an ejection fraction classification. Eur Heart J 2019;40:2155-63.

33. Farmakis D, Simitsis P, Bistola V, et al. Acute heart failure with mid-range left ventricular ejection fraction: clinical profile, inhospital management, and short-term outcome. Clin Res Cardiol 2017;106:359-68

34. Bhambhani V, Kizer JR, Lima JAC, et al. Predictors and outcomes of heart failure with mid-range ejection fraction. Eur $\mathrm{J}$ Heart Fail 2018;20:651-9.
35. Lyu S, Yu L, Tan H, et al. Clinical characteristics and prognosis of heart failure with mid-range ejection fraction: insights from a multi-centre registry study in China. BMC Cardiovasc Disord 2019;19:209.

36. Hsich EM, Grau-Sepulveda MV, Hernandez AF, et al. Relationship between sex, ejection fraction, and B-type natriuretic peptide levels in patients hospitalized with heart failure and associations with in-hospital outcomes: findings from the Get With The Guideline-Heart Failure Registry. Am Heart J 2013;166:1063-71.

37. Tromp J, Khan MA, Klip IT, et al. Biomarker profiles in heart failure patients with preserved and reduced ejection fraction. J Am Heart Assoc 2017;6:e003989.

38. Prastaro M, D'Amore C, Paolilo S, et al. Prognostic role of transthoracic echocardiography in patients affected by heart failure and reduced ejection fraction. Heart Fail Rev 2015;20:305-16.

39. Sani MU, Davison BA, Cotter G, et al. Echocardiographic predictors of outcome in acute heart failure patients in subSaharan Africa: insights from THESUS-HF. Cardiovasc J Afr 2017;28:60-7.

40. Sliwa K, Davison BA, Mayosi BM, et al. Readmission and death after an acute heart failure event: predictors and outcomes in sub-Saharan Africa: results from the THESUS-HF registry. Eur Heart J 2013;34:3151-9. 